\title{
Spectroscopy of the double-lined dwarf nova AT Ara ${ }^{\star}$
}

\author{
A. Bruch ${ }^{\star \star}$ \\ Laboratório Nacional de Astrofísica, CP 21, 37500-000 Itajubá - MG, Brazil \\ Received 6 November 2001 / Accepted 3 July 2003

\begin{abstract}
The first time-resolved spectroscopic observations of the dwarf nova AT Ara are presented. The system is a double lined spectroscopic binary. Thus, radial velocity curves of both, emission lines from the accretion disk (in this particular case of $\mathrm{H} \alpha$ ) and of the secondary star can be measured. An orbital period of 0.37551 days is derived, placing AT Ara among the cataclysmic variables with the longest revolution periods. The $R V$-amplitudes yield a mass ratio of $q=M_{2} / M_{1}=0.79 \pm 0.09$. The spectral type of the secondary star is K2. AT Ara has an intermediate orbital inclination of $38^{\circ} \pm 5^{\circ}$. The secondary star has a mass and a radius of $M_{2}=0.42 \pm 0.10 M_{\odot}$ and $R_{2}=0.84 \pm 0.19 R_{\odot}$, respectively. It is thus significantly larger than a main sequence star of the same mass. Evolutionary calculations of cataclysmic variables suggest that the secondary is nuclearly
\end{abstract} \\ evolved to a certain degree. The distance to AT Ara is estimated to be of the order of $600 \mathrm{pc}$.
}

Key words. stars: binaries: close - stars: novae, cataclysmic variables - stars: individual: AT Ara

\section{Introduction}

Dwarf novae are well-known to be a subclass of cataclysmic variables (CVs), interacting binary stars in which a Roche-lobe filling late type star (the secondary) loses mass to a white dwarf primary, which forms - if the white dwarf does not possess a strong magnetic field - an accretion disk around the latter before the transferred mass slowly settles down onto the surface of the compact star. CVs in general exhibit a large variety of observational characteristics - albeit not all in the same individual object - many of which permit the study of particular properties of the structure of these systems, but of course only if that particular observational feature is seen. For example, the presence of eclipses (of the primary, including the accretion disk, by the secondary) allows a detailed study of the disk structure via eclipse tomography. Similarly, the properties of the secondary star can best be studied in systems with a comparatively long orbital period ( $~ \approx 6 \mathrm{~h}$ ) because in short orbital period systems its luminosity is so small that it is completely drowned by the primary and the accretion disk, at least in the optical spectral range.

Concerning the latter item, the structure of the secondary star, there appears to be an increasing interest recently. Questions focus on items such as to which extent the red dwarfs in CVs may be regarded as genuine main sequence stars (Beuermann et al. 1998), the relationship between their spectral types and their masses (Kolb et al. 2001), or the reaction of the secondary to mass loss (Stehle et al. 1996). Finding answers to such questions requires on the one hand a good

* Based on observations obtained at the Observatório do Pico dos Dias, LNA/MCT, Itajubá, Brazil.

$\star \star$ e-mail: albert@lna.br theoretical understanding of the structure of late-type low-mass stars as well as their observable properties (i.e. their spectra). The considerable progress in these fields in recent years is summarized in the reviews of Charbrier \& Baraffe (2000) and Allard et al. (1997). On the other hand reliable observations of the secondary stars in CVs are required. CVs with phase resolved spectra of the late type component are rare. The corresponding sample is restricted to a small number of long period CVs. Increasing this sample is definitely important in order to enlarge the base for comparison of the theoretical models for the secondaries in CVs with the observed properties and thus to resolve problems such as those cited above.

The dwarf nova AT Ara is a promising candidate in this respect. It was originally suspected to be a SU UMa star (Warner 1976) and as such would have a short orbital period. However, the long-term photometric behaviour studied by Bateson (1978) does not support this hypothesis since the system does not show the typical dichotomy of normal and superoutburst which characterize the SU UMa stars. Moreover, the meager spectroscopic information available about AT Ara points at a long orbital period: Vogt (1976) mentions possible $G$-band absorption, and a low resolution spectrum of Bruch (1982) clearly shows the Mg I 5167-5184 A triplet and Ca I 5262-5270 $\AA$ in absorption, indicating a considerable contribution of the secondary star to the optical light. It is surprising that in spite of these features which obviously lend AT Ara particular interest, and in spite of the relatively high brightness even during quiescence of $V \approx 14$. 9 (Vogt 1983; Bruch 1983), so far no detailed observations, neither photometric nor spectroscopic, have been published in the literature.

Here, a dedicated spectroscopic study, enriched by some photometry, is presented which confirms the nature of AT Ara 
as a double-lined CV. These observations yield not only the orbital period and the radial velocity curve of both stellar components, but also permit a detailed investigation of the secondary star spectrum, yielding a secure determination of the spectral type of the secondary and also information about the component masses and the system geometry. Thus, light is shed on some of the questions raised above.

\section{Spectroscopic observations}

Spectroscopic observations of AT Ara were performed during the nights 2000, June 30, July 1 and July 2 at the 1.6-m telescope of the Observatório do Pico dos Dias, run by the Laboratório Nacional de Astrofísica (LNA), Brazil. The telescope was equipped with a Cassegrain spectrograph. The observed spectral range ( 5818-6625 $⿱$ A) encompasses the He I $5876 \AA$ and $\mathrm{H} \alpha$ emission lines as well as numerous absorption lines suitable for a detailed analysis of the late type component of AT Ara. Integration times were 15 min throughout. A narrow slit was used in order to enable radial velocity measurements. This, together with non-photometric weather condition, made it impossible to perform a spectrophotometric calibration. However, observations of the standard star LTT 7379 (Hamuy et al. 1992) permitted to roughly determine the instrumental response function and to put the spectra on an approximate relative flux scale. A Ne-Ar comparison lamp for wavelength calibration was exposed after every second spectrum of AT Ara.

The spectra were recorded on a thin, back-iluminated SITeSI003AB CCD. The basic reduction of these data, including bias-subtraction, flat-fielding, optimized spectral extraction (Horne 1986) and wavelength calibration were performed within IRAF, while the MIRA software package (Bruch 1993) was used for the further analysis of the data. A total of 56 spectra were recorded, however, most of them during the first and third night. On 2000, July 1 unfavourable weather conditions permitted only to obtain two spectra. The instrumental setup yielded a dispersion of $0.79 \AA$ pixel $^{-1}$, while a spectral resolution (derived from the $F W H M$ of the 13 emission lines, each of them measured in all comparison spectra and distributed over the entire spectra range) was $R=2.07 \pm 0.11 \AA$.

\section{Spectrum, radial velocity curve and orbital period}

\subsection{Characterization of the spectrum}

The main features seen in the individual spectra of AT Ara are a prominent $\mathrm{H} \alpha$ emission and a somewhat fainter emission of He I $5876 \AA$. Apart from these spectral characteristics which are normal in quiescent dwarf novae, AT Ara exhibits also numerous absorptions lines, confirming the earlier observations of Vogt (1976) and Bruch (1982) of a significant contribution of the secondary to the optical spectrum. These lines readily permit to classify the red component as an early K dwarf.

The upper and lower panels of Fig. 1 show the mean spectrum of AT Ara in the rest frame of the emission and absorption lines, respectively, where the radial velocity measurements

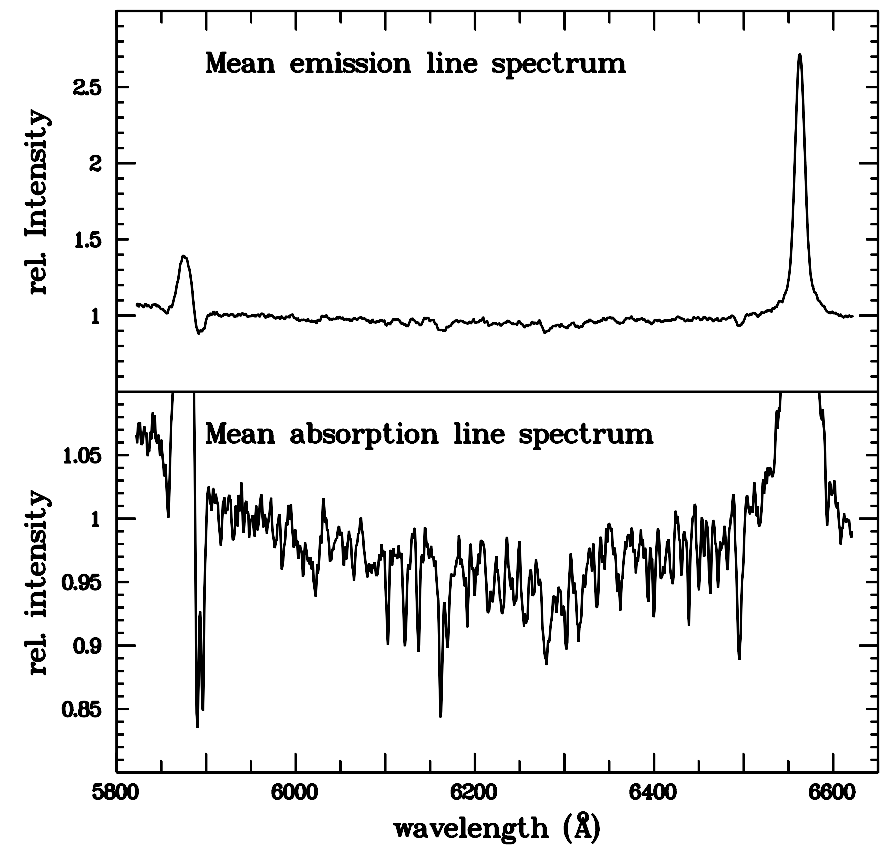

Fig. 1. Mean spectrum of AT Ara in the reference frame of the emission lines (top) and absorption lines (bottom).

to be described below have been used to adjust the individual spectra. There is no sign for structure such as a double peak in the emission lines as is often (albeit by far not always!) seen in other CVs (see e.g. Honeycutt et al. 1987). The absorption line spectrum is dominated by strong $\mathrm{Na} \mathrm{D}$ but also contains numerous other lines typical for an early K type star. A more accurate determination of the spectral type is postponed to Sect. 4.1.

\subsection{The orbital period and the radial velocity curve}

The measurement of the radial velocity $(R V)$ curve and the orbital period is the base of any dynamical study of a CV. In order to measure the $R V$ of the absorption lines the spectral region between $\sim 5900-6500 \AA$ was selected. This excludes the strong $\mathrm{Na} \mathrm{D}$ lines which may be distorted by the adjacent He I $5876 \AA$ emission and thus may introduce errors into the $R V$ measurements. A cross-correlation technique was used to determine the radial velocities of the absorption lines: As will be detailed in Sect. 4.1 the spectral type of the late type component of AT Ara is K2. Therefore, a high resolution spectrum of the K2 V star HD 4628 (Soubiran et al. 1998) was retrieved from the SIMBAD data base in order to serve as a template for cross-correlations with the AT Ara spectra. It was convolved with a Gaussian of FWHM of $2.1 \AA$ (see Sect. 2) to degrade its resolution to the intrinsic resolution of the latter. After rescaling the individual spectra and the template to a logarithmic wavelength scale the cross-correlation functions (CCFs) were calculated. Fitting the peaks of the CCFs with high order polynomials yielded the wavelength shifts between the template and each spectrum and then the $R V$ difference. Using the $R V$ of the template of $-10.31 \mathrm{~km} \mathrm{~s}^{-1}$ (Soubiran et al. 1998) the heliocentric $R V \mathrm{~s}$ of the AT Ara spectra as listed in Table 1 together 
Table 1. Heliocentric radial velocities of AT Ara

\begin{tabular}{|c|c|c|}
\hline $\begin{array}{c}\text { HJD } \\
(2441700+)\end{array}$ & $\begin{array}{l}R V(\mathrm{H} \alpha) \\
\left(\mathrm{km} \mathrm{s}^{-1}\right)\end{array}$ & $\begin{array}{c}R V \text { (abs. lines) } \\
\left(\mathrm{km} \mathrm{s}^{-1}\right)\end{array}$ \\
\hline 26.4374 & -64.6 & -14.0 \\
\hline 26.4496 & -42.6 & 2.6 \\
\hline 26.4603 & -10.5 & -12.2 \\
\hline 26.4736 & 3.1 & -31.8 \\
\hline 26.4842 & 26.5 & -41.4 \\
\hline 26.4964 & 15.5 & -85.3 \\
\hline 26.5212 & 34.3 & -118.9 \\
\hline 26.5319 & 42.5 & -120.0 \\
\hline 26.5441 & 58.6 & -126.8 \\
\hline 26.5548 & 57.2 & -136.6 \\
\hline 26.5668 & 52.8 & -143.7 \\
\hline 26.5775 & 53.1 & -109.5 \\
\hline 26.5899 & 52.0 & -106.5 \\
\hline 26.6006 & 56.8 & -93.1 \\
\hline 26.6129 & 56.8 & -94.1 \\
\hline 26.6236 & 21.7 & -61.0 \\
\hline 26.6356 & -1.7 & -46.2 \\
\hline 26.6464 & 11.2 & -41.7 \\
\hline 26.6586 & -23.9 & -5.1 \\
\hline 26.6693 & -36.7 & -6.8 \\
\hline 26.6813 & -42.4 & 21.1 \\
\hline 26.6920 & -96.2 & -67.7 \\
\hline 26.7039 & -66.2 & 66.2 \\
\hline 26.7146 & -84.7 & 45.4 \\
\hline 26.7267 & -103.2 & 78.6 \\
\hline 26.7374 & -88.7 & 74.2 \\
\hline 26.7495 & -93.8 & 78.9 \\
\hline 26.7602 & -97.9 & 77.5 \\
\hline 26.7725 & -74.2 & 74.4 \\
\hline 26.7833 & -82.2 & 60.3 \\
\hline 27.5985 & -12.8 & -11.1 \\
\hline 27.6341 & 82.5 & -91.7 \\
\hline 28.5127 & 27.9 & -25.9 \\
\hline 28.5267 & -11.3 & -12.5 \\
\hline 28.5374 & -8.8 & 41.7 \\
\hline 28.5494 & -26.4 & 1.8 \\
\hline 28.5601 & -27.7 & 27.8 \\
\hline 28.5731 & -50.8 & 58.8 \\
\hline 28.5838 & -52.6 & 51.8 \\
\hline 28.5958 & -66.8 & 53.3 \\
\hline 28.6064 & -72.0 & 72.5 \\
\hline 28.6184 & -63.3 & 107.6 \\
\hline 28.6291 & -82.2 & 46.8 \\
\hline 28.6416 & -91.5 & 44.0 \\
\hline 28.6523 & -93.6 & 68.4 \\
\hline 28.6644 & -62.8 & -25.0 \\
\hline 28.6750 & -67.8 & -108.0 \\
\hline 28.6876 & -29.5 & 16.7 \\
\hline 28.6983 & -22.0 & 33.2 \\
\hline 28.7103 & -5.9 & 35.0 \\
\hline 28.7210 & 27.4 & -18.3 \\
\hline 28.7345 & 49.4 & -47.4 \\
\hline 28.7451 & 47.1 & -47.1 \\
\hline 28.7577 & 56.6 & -72.6 \\
\hline 28.7685 & 53.9 & -115.3 \\
\hline 28.7807 & 81.1 & -91.4 \\
\hline
\end{tabular}

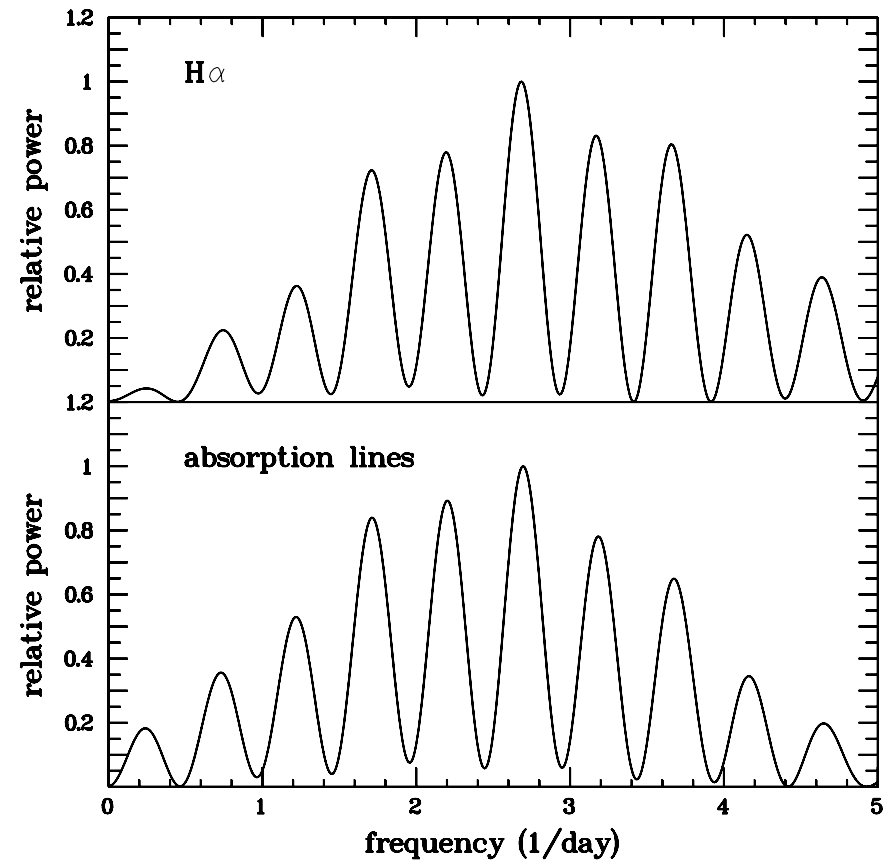

Fig. 2. Power spectra of $\mathrm{H} \alpha$ (top) and absorption line radial velocities (bottom).

with the heliocentric Julian Dates at midexposure were then calculated.

The algorithm of Deeming (1975) was used to calculate the power spectrum of the $R V$ data. It is shown in the lower frame of Fig. 2 which exhibits a strong alias structure with a dominant peak at $\approx 2.695$ cycles day $^{-1}$. The corresponding period was used as a start value for a least squares fit of a sinecurve to the $R V$ data (disregarding three discrepant $R V$ values at HJD $2441726.6920,2441728.6644$ and 2441728.6750$)$. The resulting period is 0.37612 days or or $9^{\mathrm{h}} 2^{\mathrm{min}}$.

The presence of the alias peaks in the power spectrum raises the question whether the chosen frequency is the correct one or if the true frequency is one of the aliases. Therefore least square sine fits were also calculated with start values for the periods corresponding the alias frequencies. Apart from a slightly worse fit to the data of June 30 and July 2, one of the two data points of July 1 is completely out of phase in these cases ${ }^{1}$. It may therefore safely be concluded that the original choice of the frequency is correct.

Two different techniques were adopted in order to measure the $R V$ of the emission line component: (1) since the structure of the $\mathrm{H} \alpha$ emission is simple, Gaussian profiles can excellently be fitted to the line. The peak of these fits yields the location of the line centre and thus the $R V$. (2) in another approach, the straight mean of all spectra, restricted to the wavelength range centred on $\mathrm{H} \alpha(6500-6620 \AA)$ was calculated and used as a template to calculate $\mathrm{CCFs}$ for the individual spectra. The peak of the CCFs was fitted with a high order polynomial in order to yield its exact location. These positions were used to shift the individual spectra to a common rest frame. In a second

\footnotetext{
1 The second point is close to the $\gamma$-velocity and close to either phase 0.0 or 0.5 so that it is always close to either the rising or the declining branch of the sine-curve.
} 
iteration step the mean of the latter spectra was calculated which then served as an improved template for a crosscorrelation with the original spectra. The peaks of the CCFs, determined in the same way as before, were finally used to calculate the $R V \mathrm{~s}$ of the individual spectra.

Both methods yielded consistent results. Therefore, in the following, the mean $R V$ values are regarded. The power spectrum of the $R V$ data is shown in the upper frame of Fig. 2. It is consistent with the power spectrum of the absorption line $R V \mathrm{~s}$. The period corresponding to the frequency of the dominant peak was used as a start value for a least squares fit of a sine-curve to the $R V$ data resulting in a best fit period of 0.37490 days or $9^{\mathrm{h}} 0^{\mathrm{min}}$. The periods corresponding to the alias peaks in the power spectrum can be rejected using the same argument as in the case of the absorption lines.

The periods derived from both, the absorption and the emission lines are thus consistent with each other. Their mean of $0.37551 \pm 0.00086$ days or $9^{\mathrm{h}} 0^{\min } 44^{\mathrm{s}} \pm 74^{\mathrm{s}}$ is adopted as the orbital period of AT Ara. The error of the period translates into a phase error of 0.014 across the entire time base of the observations. In view of the scatter of the data points this would scarcely be noticable. Therefore, the formal error should be regarded as a lower limit to the true error.

It is well known that the radial velocity measured from the emission lines of a $\mathrm{CV}$ may depend on the part of the lines which is used for the measurement. The emission may originate in a large part of the accretion disk and may not necessarily be azimuthally symmetric around its centre (i.e. the white dwarf). Therefore, it is dangerous to use $R V$ measurements of the emission lines at face value in an analysis of the dynamics of the system. Care must be taken to sample those parts of the emission lines which originate as closely as possible to the white dwarf if the motion of the compact primary is to be measured. It is therefore common practice to use the double Gaussian convolution method (Schneider \& Young 1980) in connection with the diagnostic diagram introduced by Shafter (1983), hereafter referred to as SYS method, to measure the $R V$ of the emission lines as a function of the distance from the line centre. Since the line wings are due to the high rotational velocity of the accretion disk close to the white dwarf, their $R V$ is expected to reflect best the motion of the primary. The diagnostic diagrams then help to decide at which distance from the line centre noise starts to dominate over the signal and the $R V \mathrm{~s}$ thus become unreliable.

The SYS method was applied to the $\mathrm{H} \alpha$ emission of AT Ara after the heliocentric correction had been applied to the wavelength scale of each spectrum. Radial velocity curves were determined for values $a$ of the separation between the centre of the double Gaussians between 2 and $13.8 \AA$ in steps of $0.2 \AA$. The parameter $\sigma$ of the Gaussians was chosen to be $2 \AA$. For each $a$ a sine curve with a period fixed at the values determined above was fitted to the $R V$ curve, the free parameters being the amplitude $K$, the systemic velocity $\gamma$ and the phase $\phi_{0}$. These quantities, together with the relative error $\sigma_{K} / K$ of the amplitude, as a function of the separation $a$ of the Gaussians, constitute the diagnostic diagram which is shown in Fig. 3.

At large values of the Gaussian separation $a$ the relative error $\sigma_{K} / K$ of the amplitude of the best fit sine curve to the radial

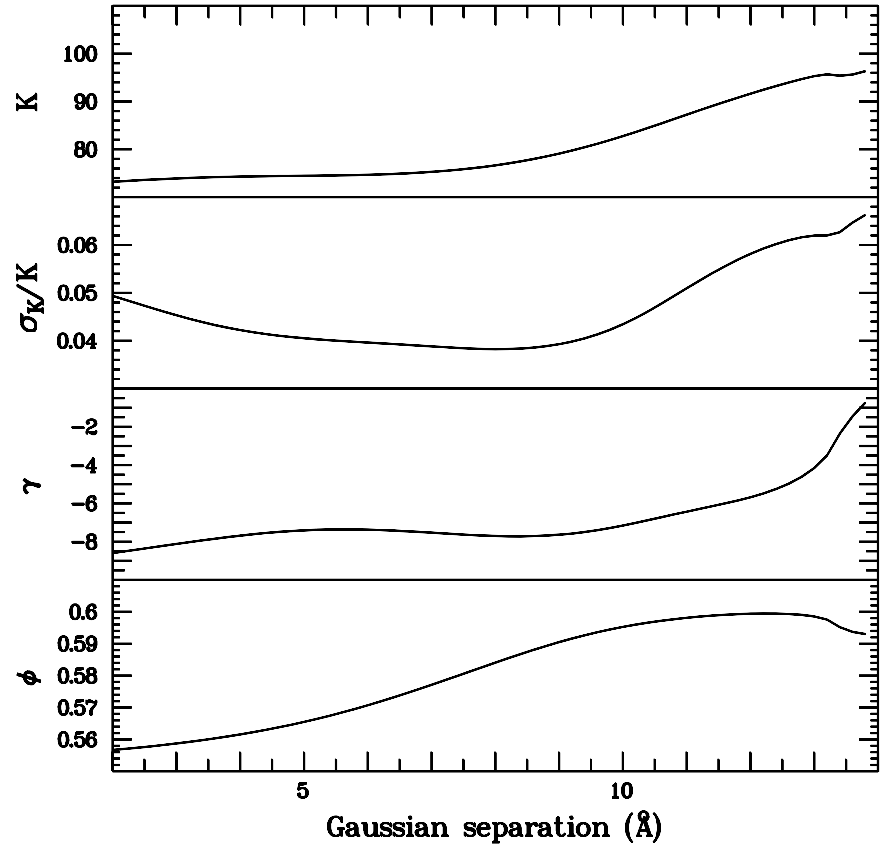

Fig.3. Diagnostic diagram resulting from radial velocity measurements of the $\mathrm{H} \alpha$ emission line using the double Gaussian convolution method. For details, see text.

Table 2. Orbital parameters of AT Ara.

\begin{tabular}{|c|c|c|}
\hline$P$ (days): & \multicolumn{2}{|c|}{$0.37551 \pm 0.00086$} \\
\hline$T_{0}(\mathrm{HJD}):$ & 2451726.465 & \pm 0.002 \\
\hline$K_{1}\left(\mathrm{~km} \mathrm{~s}^{-1}\right)$ & 78.9 & \pm 3.1 \\
\hline$K_{2}\left(\mathrm{~km} \mathrm{~s}^{-1}\right):$ & 99.5 & \pm 3.2 \\
\hline$\gamma_{\mathrm{emi}}\left(\mathrm{km} \mathrm{s}^{-1}\right)$ & -7.4 & \pm 2.1 \\
\hline$\gamma_{\mathrm{abs}}\left(\mathrm{km} \mathrm{s}^{-1}\right)$ : & -21.3 & \pm 2.2 \\
\hline
\end{tabular}

velocity curve rises rapidly. The values of $K, \gamma$ and $\phi_{0}$ corresponding to a values of $a$ immediately before this rise are generally considered to reflect best the motion of the white dwarf. In the present case, the $\sigma_{K} / K$-curve starts to increase close to $a=9 \AA \hat{=} 410 \mathrm{~km} \mathrm{~s}^{-1}$. The amplitude, systemic velocity, epoch of zero-crossing from negative to positive $R V \mathrm{~s}$ (calculated from the phase $\phi_{0}$ ) and the $\gamma$ velocity for this $a$ are listed together with the adopted orbital period in Table 2 . Note that the errors are purely formal fit errors and definitely underestimate the true errors in view of a certain arbitrariness in the choice of the optimal values of the Gaussian separation. The $R V$ values of $\mathrm{H} \alpha$ of the individual spectra are listed in Table 1.

The $R V$ amplitude and the corresponding $\gamma$ velocity determined through a sine-fit to the absorption line $R V \mathrm{~s}$ are also listed in Table 2. Both, the absorption and emission line data, folded on the orbital period, are plotted together with the best fit sine curves in Fig. 4.

\subsection{The line structure}

In order to investigate the line structure a greyscale image of the spectrum of AT Ara as a function of orbital phase has been 


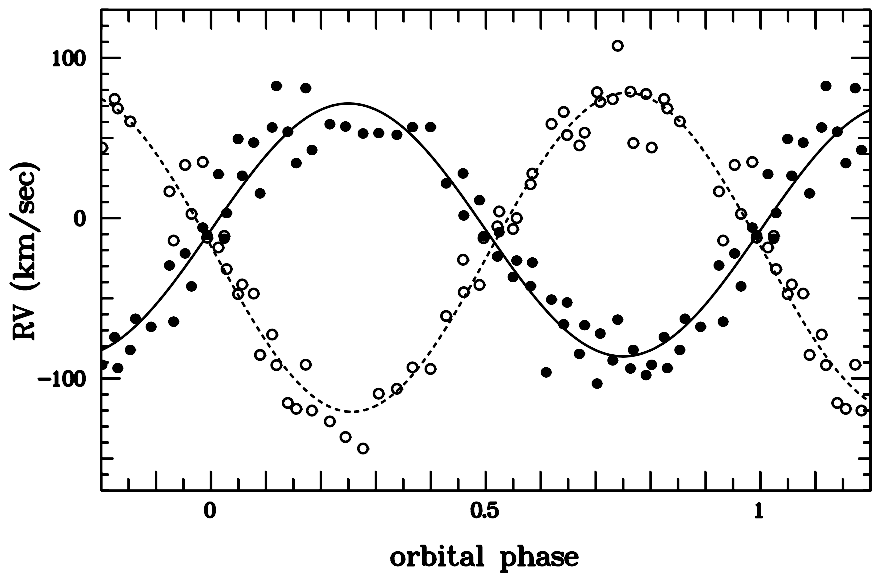

Fig. 4. Radial velocity curve of the $\mathrm{H} \alpha$ emission line (filled circles) and absorption lines (open circles), folded on the orbital period, together with the best fit sine-curves (solid and dashed lines, respectively).

constructed by first normalizing all spectra to the continuum and than stacking them in phase. The resulting image is shown in Fig. 5.

The antiphased radial velocity variations of all absorption and emission lines are readily visible. There appear to be slight phase-dependent variations in the absorptions line strength. However, care must be taken not to overinterpret the data which cover only about two orbital cycles. Variations in the equivalent width of the lines caused by a variable veiling due to flickering or night-to-night variations could therefore well mimic phasedependences of the line strength. Therefore, they will not be interpreted further here. The same holds true for the $\mathrm{H} \alpha$ emission line which appear somewhat knotty as a function of phase (not well visible in the figure because in the reproduction $\mathrm{H} \alpha$ is "overexposed"). The equivalent width of this line has been measured in the individual spectra. While no phase dependent variations are apparent, the mean values rose from $27 \pm 2 \AA$ in the first to $33 \pm 5 \AA$ in the third observing night, confirming night-to-night variability. At no phase the $\mathrm{H} \alpha$ emission exhibits a wavelength dependent structure such as double peaks or other indications of multiple component emissions. Fits of a Gaussian to the line profiles yielded a mean value of the FWHM of $13.7 \pm 0.7 \AA$ without indications for night-to-night changes.

\section{The absorption line spectrum}

\subsection{The spectral class of the late type component}

In order to determine the spectral class of the secondary star of AT Ara high resolution spectra of G- and K-stars (Soubiran et al. 1998) were retrieved from the SIMBAD data base. They were convolved with a Gaussian of $F W H M$ of $2.1 \AA$ in order to degrade their resolution to the intrinsic resolution of the AT Ara spectra (see Sect. 3.2).

The intensity ratio between the Ca I $\lambda \lambda$ 6103-6170 $\AA$ and Fe I $\lambda 6137 \AA$ lines is a good indicator of the spectral type. In Fig. 6 (left frame) the spectra of 6 main sequence stars with spectral types between $\mathrm{G} 8 \mathrm{~V}$ and $\mathrm{K} 5 \mathrm{~K}$ are plotted together with the mean spectrum of AT Ara. Of course, the absorption lines in AT Ara are shallower than those of the standard stars because of veiling, and they appear to be somewhat broader. The latter effect can be interpreted as being due to rotation (see Sect. 4.2). For spectral types of K1 V and earlier, Ca I $\lambda 6103 \AA$ as well as Fe I $\lambda 6137 \AA$ are slightly stronger that Ca I $\lambda 6122 \AA$, while for spectral types of $\mathrm{K} 3 \mathrm{~V}$ and later the opposite is true. For $\mathrm{K} 2 \mathrm{~V}$ all these lines have about the same strength. There is no appreciable difference in the strength of the mentioned lines in the AT Ara spectrum, permitting to classify the secondary star as a K2 star.

As for the luminosity class, the spectral range around $\lambda 6450 \AA$ contains good indicators. In the right frame of Fig. 6 the spectrum of AT Ara is plotted together with spectra of $\mathrm{K} 1$ stars of different luminosity classes (no suitable K2 star spectrum was available). It is seen that for stars above the main sequence the line Ca I $\lambda 6450 \AA$ is stronger that the neigbouring lines of $\mathrm{Ca} I \lambda$ 6439, $6462 \AA$ and Fe I $\lambda 6431 \AA$. In the (somewhat noisy) AT Ara spectrum Ca I $\lambda 6450 \AA$ is even weaker than the neighbouring lines, excluding a luminosity class higher than $\mathrm{V}$.

Thus, from the point of view of spectral classification there is no indication for an evolved secondary star in AT Ara (but see Sect. 6).

\subsection{Veiling and line broadening}

Two effects alter the shape and the depth of the absorption lines in a CV. Veiling, defined as the fractional contribution of the primary to the total light, makes the lines shallower. At the same time, the high spin velocity due to the bound rotation of the secondary star broadens the absorption lines. Quantifying the latter effect and thus measuring the projected rotational velocity $v_{\mathrm{r}} \sin i$ is important since it is a function only of the mass ratio $q=M_{2} / M_{1}$ of the system and the measured $R V$ amplitude, permitting thus a largly independent determination of $q$. Since both, veiling and line-broadening, affect the same observable quantity, namely the line profile, they will be determined simultaneously here.

To this end, two spectral region with well defined absorption lines are regarded, (1) $\lambda \lambda$ 6074-6205 $\AA$, and (2) $\lambda \lambda 6353-$ $6509 \AA$. The mean absorption spectrum of AT Ara (normalized to the contiuum) in these regions was compared to the standard K2 V spectrum. First, the cross-correlation function of AT Ara and the $\mathrm{K} 2 \mathrm{~V}$-star was calculated for both regions. The peak of the CCF was fitted with a high order polynomial. The position of the peak yielded a correction which was applied to the wavelength scale of the standard star in order to put both stars on the same wavelength zeropoint.

In order to simulate the rotation of the secondary star, the standard star spectrum (which had already been smoothed to meet the intrinsic resolution of the AT Ara spectra; see Sect. 4.1) was convolved with a Gaussian of width $\sigma_{1}$. Veiling was simulated by adding a constant $v$ to the comparison spectrum and then dividing the spectrum by $1+v$. A grid of such spectra with $\sigma_{1}$ and $v$ varying independently in the range of 0.0-2.0 in steps of 0.1 was calculated. Finally, the variance of the difference between the AT Ara spectrum and the 


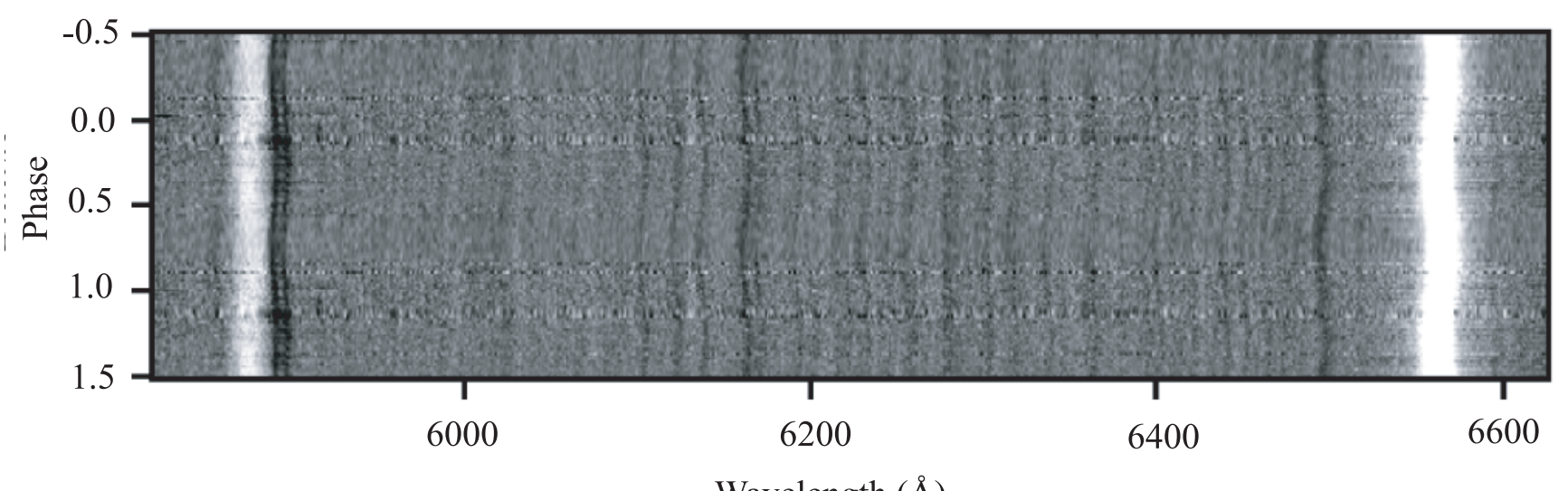

Fig. 5. Greyscale image of the spectrum of AT Ara as a function of orbital phase.

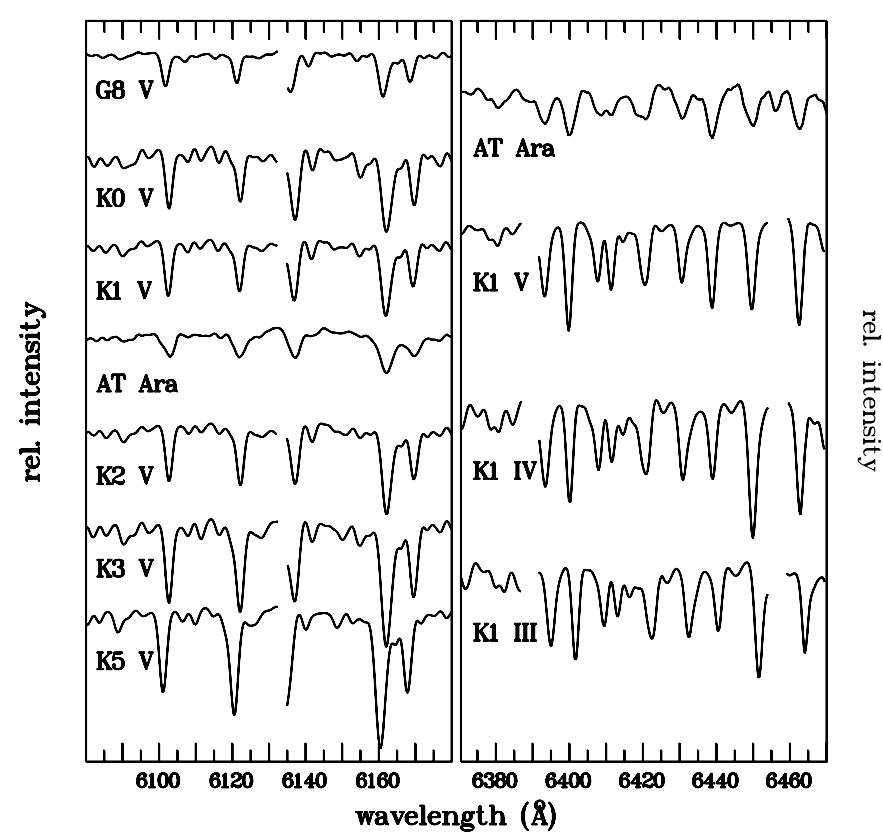

Fig. 6. Comparison of the spectrum of AT Ara within selected spectral ranges with the spectra of late type standard stars of different spectral types (left) and luminosity classes (right).

processed comparison star spectra was determined which should have a minimum at the optimal values for $\sigma_{1}$ and $v^{2}$. The variance is shown as a function of $\sigma_{1}$ and $v$ in Fig. 7 for regions 1 (left) and 2 (right). Minimum values for the variance are assumed for $\sigma_{1}=1.1, v=0.5$ in region 1 , and $\sigma_{1}=1.0$, $v=0.6$ in region 2 . Thus, there is no appreciable inconsistency between the two regions, and the mean (i.e. $\sigma_{1}=1.05$, $v=0.55$ ) will be adopted as the final value. Since the $\mathrm{S} / \mathrm{N}$ ratio of the spectra is not known quantitatively, it is not possible to assign an objective error in a statistically meaningful sense

\footnotetext{
${ }^{2}$ In principle, not only should the variance assume a minimum, but the mean value of the difference should simultaneously attain a value of 0 . This, however, is often impeded by slight differences in the normalization of the two spectra. Therefore, the latter criterium cannot be employed here.
}

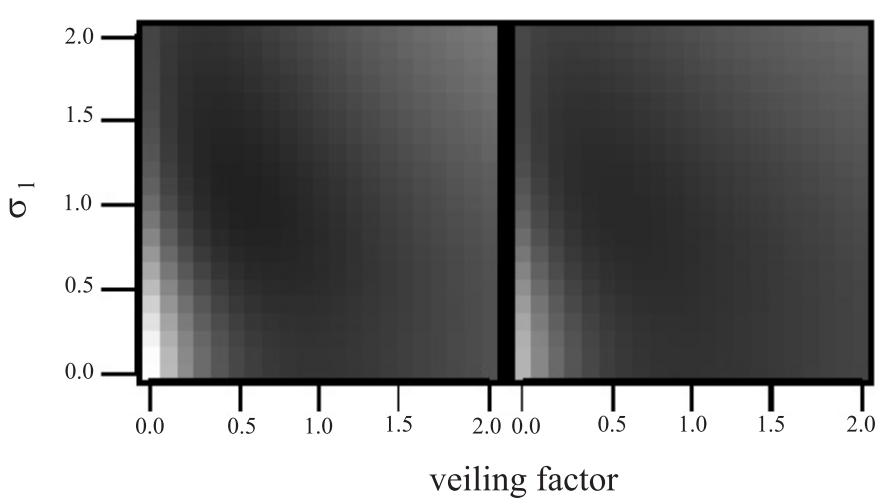

Fig. 7. The variance between the mean AT Ara absorption line spectrum and the spectrum of a $\mathrm{K} 2 \mathrm{~V}$ standard star as a function of the adopted values for the veiling factor $v$ and Gaussian parameter $\sigma_{1}$ used to broaden the standard spectrum in order to simulate stellar rotation, for the spectral regions $\lambda \lambda$ 6074-6205 $\AA$ (left) and $\lambda \lambda$ 6353-6509 (right).

to these values. As a lower limit to the error, the difference between the mean value and the two individual measurements of $\sigma_{1}$ and $v$ (i.e. $\sigma_{\sigma_{1}}=0.05$ and $\sigma_{v}=0.05$ ) are adopted here. In order to determine the dependency of $\sigma_{1}$ and $v$ on the assumed value of the spectral resolution, the calculations were repeated with a resolution deviating from the nominal resolution by $\pm 1 \sigma_{R}$ and $\pm 4 \sigma_{R}$, where $\sigma_{R}$ is the standard deviation as quoted in Sect. 2. While in the first of theses cases the difference is of the order of 0.04 in $\sigma_{1}$ and 0.02 in $v$ and thus smaller than our adopted error, in the second (exaggerated) case the difference grows to $\sim 0.25$ in $\sigma_{1}$ but at 0.04 remains small in $v$.

\subsection{The projected rotational velocity of the secondary star}

In order to extract useful physical information from the measured line width $\sigma_{1}$, the relationship between $\sigma_{1}$ and the projected rotational velocity $v_{\mathrm{r}} \sin i$ must be established, where $v_{\mathrm{r}}$ is the equatorial velocity of the secondary star (bound rotation is assumed) and $i$ is the inclination of the rotation axis with 
Table 3. Journal of photometric observations.

\begin{tabular}{lcccccc}
\hline \hline Date & $\begin{array}{c}\text { JD } \\
\text { (from - to })\end{array}$ & $\begin{array}{c}\text { Total time } \\
\text { base } \\
(2450000+)\end{array}$ & $\begin{array}{c}\text { Number of } \\
\text { integrations }\end{array}$ & $\begin{array}{c}\text { Time } \\
\text { resolution } \\
(\mathrm{min})\end{array}$ & $\begin{array}{c}\text { Pass- } \\
\text { band }\end{array}$ & $\Delta m$ \\
\hline 1999, Aug. 30 & $1421.414-.619$ & 293 & 259 & 69 & $R$ & $1.36 \pm 0.08$ \\
1999, Aug. 31 & $1422.402-.633$ & 333 & 284 & 69 & $R$ & $1.52 \pm 0.07$ \\
1999, Sep. 1 & $1423.410-.609$ & 286 & 219 & 69 & $R$ & $1.64 \pm 0.05$ \\
2001, June 30 & $2091.404-.790$ & 556 & 6168 & 5 & white & $1.55 \pm 0.06$ \\
2001, July 1 & $2092.397-.709$ & 450 & 4558 & 5 & white & $1.52 \pm 0.05$ \\
\hline
\end{tabular}

respect to the line-of-sight (thought to be equal to the orbital inclination of the system).

To do so, model calculations were performed. The intrinsic absorption line profile is assume to be a Voigt profile, using a damping constant of $\gamma=10^{9} \mathrm{~s}^{-1}$ and an atmospheric temperature of $4900 \mathrm{~K}$ (but note that the results are quite insensitive to these details). The stellar surface was thought to be composed of a grid of surface elements each of which has a radial velocity with respect to the observer depending upon its location on the star, the inclination angle $i$, and the equatorial rotational velocity $v_{\mathrm{r}}$. The contributions to the total profile of all individual surface elements visible by the observer are then added up, taking into account the radial velocity, the geometrical foreshortening of the area, and the limb darkening. For the latter, a value of 0.588 was derived via an interpolation in the tables of Wade \& Rucinski (1985), adopting the above mentioned temperature and a surface gravity of $\log g=4.2\left(M=0.42 M_{\odot}\right.$; $R=0.83 R_{\odot}$; see Sect. 6.2). The resulting profile was convolved with a Gaussian with $F W H M$ of $2.1 \AA$ in order to take the instrumental resolution into account. Line profiles were calculated for a grid of parameters $i\left(0^{\circ}-90^{\circ}\right.$ in steps of $\left.5^{\circ}\right)$ and $v_{\mathrm{r}}$ $\left(40 \mathrm{~km} \mathrm{~s}^{-1}-300 \mathrm{~km} \mathrm{~s}^{-1}\right.$ in steps of $\left.20 \mathrm{~km} \mathrm{~s}^{-1}\right)$. Each of the profiles was subjected to a Gaussian fit, yielding the value $\sigma_{1}$ required to finally establish the $v_{\mathrm{r}} \sin i-\sigma_{1}$ relation.

This relationship can excellently be fitted by a polynomial of order 3. Evaluating the polynomial for $\sigma_{1}=1.05 \pm 0.05$, the measured width of the absorption lines in AT Ara (see Sect. 4.2), yields $v_{\mathrm{r}} \sin i=69.5 \pm 14.1 \mathrm{~km} \mathrm{~s}^{-1}$.

\section{Photometric observations}

Time resolved differential CCD photometry of AT Ara was performed in five nights in 1999, August/September and 2001, June/July. The 1999 observations were carried out at the $0.6-\mathrm{m} \mathrm{B \& C}$ telescope of the LNA, using the same $\mathrm{CCD}$ as detector as for the spectroscopic observations (see Sect. 2). The 2001 observations were performed with the LNA 0.6-m Zeiss telescope which was equipped with a thin, back-illuminated CCD of type EEC CCD-02-06-1-206. It was run in a frame-transfer mode, permitting observations with practically no dead-time between integrations and thus a high time resolution. The reduction of these data was performed with the IRAF based aperture photometry routine LCURVEAP (courtesy M. Diaz). Differential magnitudes of AT Ara with respect to four comparison stars in the field were obtained.

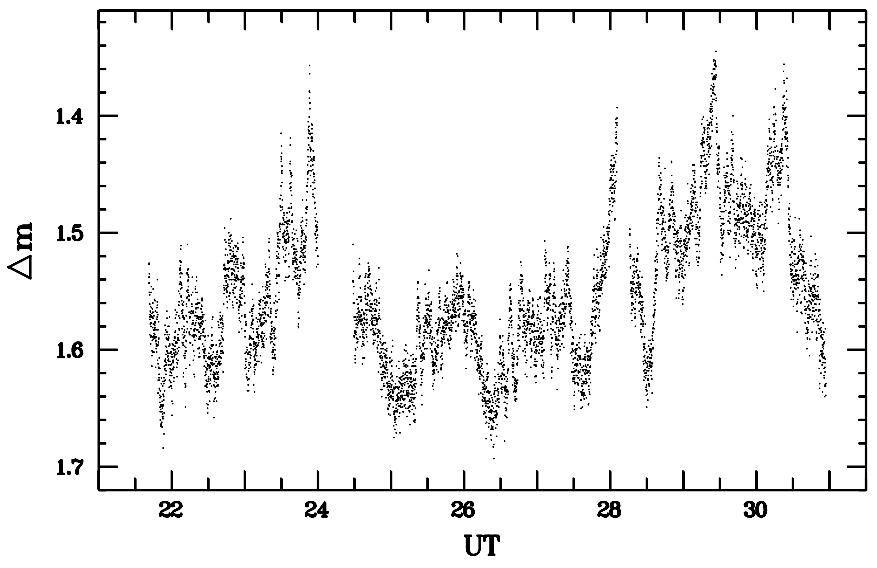

Fig. 8. Light curve of AT Ara on 2001, June 30/July 1. The time resolution is $5 \mathrm{~s}$.

Weather conditions were not perfect. But the constancy of the magnitude differences between the comparison stars showed that the differential photometry is reliable. A journal of the photometric observation is given in Table 3 . The individual columns list (1) the civil date (at the start of the observations), (2) the Julian Date of start and end, (3) the total time base of each light curve, (4) the time resolution, (5) the number of exposures, (6) the photometric passband, and (7) the mean magnitude difference between AT Ara and the principal comparison star. The standard deviation of the latter is mainly due to the flickering activity of the dwarf nova.

As an example the longest of the light curves which covers more than an entire orbit is shown in Fig. 8. The general characteristics of the other ones are similar. The light curve is dominated by flickering occuring on the time scale of minutes which is superposed by flaring on hourly time scales (see Bruch 2000 for a discussion of the distinction between flickering and variations on longer time scales). Regarding all light curves together it is seen that the latter does not repeat regularly. In particular, the long time base of the light curves shows that there is no evidence for variability with the orbital period, suggesting that the orbital inclination for AT Ara is not high.

\section{Discussion}

The numerous hazards of taking the parameters of the radial velocity curves of the emission and absorption lines of CVs 
at face value for deriving dynamical quantities of a CV system are well known. In particular, it is often observed that the phase difference between absorption and emission lines differs from 0.5 , indicating that the emission line radial velocities do not reflect well the motion of the white dwarf. Moreover, in the case of strong illumination of the secondary by the primary, the absorption line $R V \mathrm{~s}$ may not represent the motion of the centre of mass of the secondary.

In the present case these concerns do not apply strongly. The sine fit to the $R V$ curves of emission and absorption lines shows a phase difference of $0.506 \pm 0.007$. Thus, the emission appears to be well centred on the white dwarf (unless due to some conspiracy it is centred on the line connecting the components but before or behind the primary!). Illumination effects are not known to be strong in long-period quiescent dwarf novae. Moreover, the relative strength of the absorptions lines used to measure the $R V$ of the secondary do not vary strongly with spectral type (thus temperature) for late G-intermediate $\mathrm{K}$ type stars, meaning that even in the presence of an illumination effect the adopted technique to measure the $R V \mathrm{~s}$ samples the same lines and give them comparable weight independent of orbital phase.

Therefore, it will be assumed here that the $R V$ curves reflect well the motion of the primary and secondary component.

\subsection{The mass ratio}

The amplitudes of the radial velocity curves of the two components of AT Ara yield immediately $q=M_{2} / M_{1}=K_{1} / K_{2}=$ $0.79 \pm 0.09$, where the error is propagated from the formal errors of the sine fits to the $R V$ curves. Assuming bound rotation, the measured value of $v_{\mathrm{r}} \sin i$ of the secondary star opens a (partially) independent way to measure the mass ratio. In a semidetached binary system, these quantities obey the functional relationship (see e.g. Friend et al. 1990): $v_{\mathrm{r}} \sin i=K_{2}(1+q) R_{\mathrm{L}_{2}}$, where $R_{\mathrm{L}_{2}}=R_{\mathrm{L}_{2}}(q)$ is the average Roche lobe radius of the secondary which can be approximated e.g. by Eggleton's (1983) formula. Using the measured values for $v_{\mathrm{r}} \sin i$ and $K_{2}$ it is then found that $q=0.89 \pm 0.26$. Within the error margins this is consistent with the mass ratio derived from $K_{1}$ and $K_{2}$.

\subsection{Masses, dimensions and geometry}

As long as the orbital inclination $i$ of AT Ara is unkown the masses of the components cannot be determined from first principles. However, the measured radial velocity amplitude of the primary component yields the mass function which, together with the mass ratio, yields $M_{2} \sin ^{3} i=0.098 \pm 0.017 M_{\odot}$ (where the first of the two above cited values of $q$ has been used). On the other hand, the measured rotational velocity of the secondary and the radius (assuming again bound rotation) yields $R_{2} \sin i=0.51 \pm 0.10 R_{\odot}$. The relation between $M_{2}$ and $R_{2}$ which is shown as a solid line in Fig. 9, parametrized by the orbital inclination can then be constructed. Errorbars are shown for selected values of $i$. Figure 9 also shows the theoretical $M_{2}-R_{2}$ relation based on the calculations of Charbrier \& Baraffe (1997) for age 1 Gyr (dashed line) and $10 \mathrm{Gyr}$

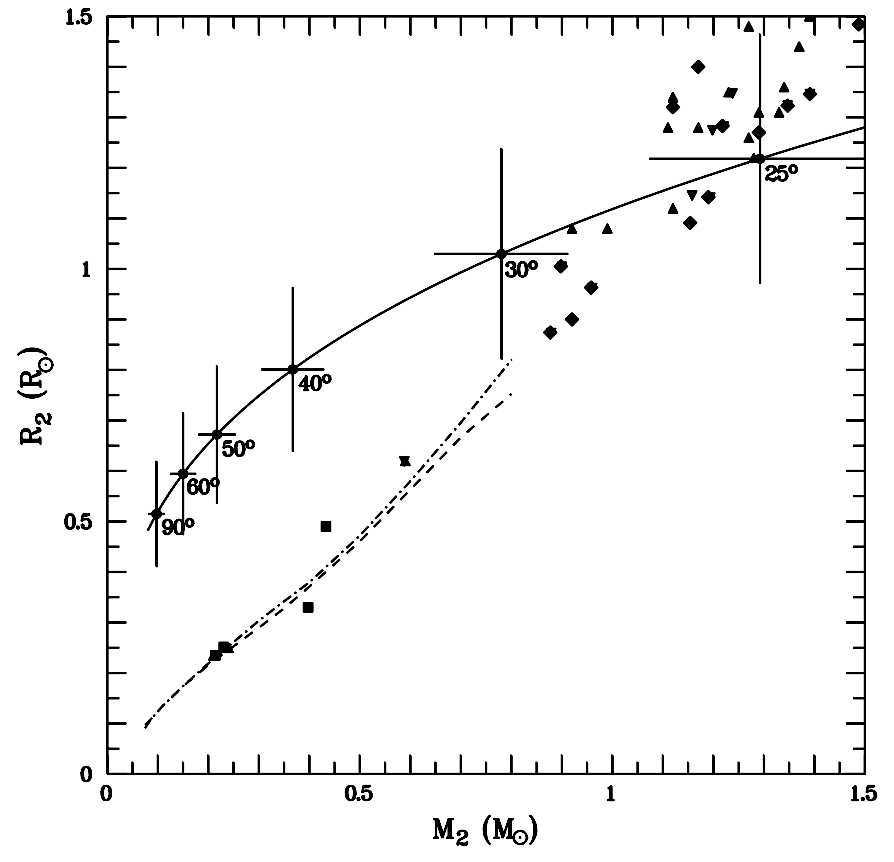

Fig. 9. Relation between mass and radius of the secondary star of AT Ara as derived from the measured mass function, mass ratio and projected rotational velocity (solid line). The graph is parametrized by the orbital inclination $i$; error bars are shown for selected values of $i$. The dashed and dashed-dotted lines are theoretical mass-radius relations for low mass stars of age $1 \mathrm{Gyr}$ and $10 \mathrm{Gyr}$, respectively, taken from Charbrier \& Baraffe (1997). The filled symbols mark the measured location of main sequence stars taken from various references (see text for details).

(dashed-dotted line). The upside-down and upside-up triangles and the diamonds, respectively, show the measured masses and radii of detached components in main sequence binaries as complied by Anderson (1991), Popper (1980) and Harmanec (1988). The squares represent recent determinations of $M$ and $R$ for low mass stars by Metcalfe et al. (1996) and Delfosse et al. (1999). If the AT Ara secondary has a low mass ( $\left.\lesssim 0.8 M_{\odot}\right)$ it lies well above the theoretical and emipircal mass-radius relationships for main sequence stars. Only for masses $M_{2} \gtrsim$ $0.8 M_{\odot}$ (implying low orbital inclinations) its radius is compatible with that of a main sequence star of the same mass.

In order to say more about the mass and the radius of the AT Ara secondary a handle on the orbital inclination is required. A tight upper limit of $M_{2}<1.14 M_{\odot}$ is given by the condition that the primary component should remain below the Chandrasekhar limit. This corresponds to $i>26^{\circ}$ and $R_{2}<1.17 R_{\odot}$.

A further, albeit weak constrain is provided by photometry: The light curves of AT Ara show no indication of an eclipse. Using the graphs in Fig. 4 of Bruch et al. (2000) it can be seen that the absence of eclipses leads to a firm upper limit of $i<70^{\circ}$ for the orbital inclination of AT Ara (no eclipse of the white dwarf) and a somewhat less firm upper limit of $i<59^{\circ}$ (no eclipse of an accretion disk with an assumed radius of $0.35 a$, where $a$ is the component separation). This constrains the parameters of the AT Ara secondary to $M_{2}>0.12 M_{\odot}$ 
and $R_{2}>0.55 R_{\odot}$ for the more stringent limit on the inclination and to $M_{2}>0.15 M_{\odot}$ and $R_{2}>0.60 R_{\odot}$ in the second case.

Combining the above derived value of $M_{2} \sin ^{3} i$ with the assumption that the accretion disk and the emission line region extend all the way down to the surface of the white dwarf enables to derive definite values of $i, M_{2}$ and $R_{2}$. The outer wings of the $\mathrm{H} \alpha$ emission line should then reflect the projected Keplerian velocity at the surface of the white dwarf. It is not easy to measure unambiguously at which wavelength the $\mathrm{H} \alpha$ emission merges into the continuum. Using the mean spectrum (in the reference frame of the primary star) shown in Fig. 1 it is estimated that the line wings extend to a distance of approximately $37 \pm 5 \AA$ from the line centre, corresponding to a projected radial velocity of about $1690 \pm 230 \mathrm{~km} \mathrm{~s}^{-1}$. Using Nauenberg's (1971) mass-radius relation for white dwarfs (assuming a mean molecular weight $\mu=2$ for simplicity) the mass $M_{1}$ of the white dwarf required for the measured projected velocity has been calculated as a function of the assumed orbital inclination $i$, yielding an $i-M_{1}$ relationship for AT Ara. Using the measured mass ratio $(q=0.79 \pm 0.09$ is adopted $)$ it can be transformed into the $i-M_{2}$ relationship shown as a dashed-dotted line in Fig. 10. The lateral dashed lines mark the error ranges which include both, the error of the total line width and of the mass ratio. On the other hand, the above derived expression $M_{2} \sin ^{3} i=0.098 \pm 0.016 M_{\odot}$ yields a second $i-M_{2}$ relationship which is shown as a solid line (again with the error range marked by lateral dashed lines) in Fig. 10. The intersection between the two graphs then yields the formal solution realized in AT Ara. The corresponding numerical values for $i$ and $M_{2}$ are summarized together with the ensuing white dwarf mass $M_{1}$ and the secondary star radius $R_{2}$ in Table 4 .

Comparing $M_{2}$ and $R_{2}$ as given in Table 4 with the graphs in 9 suggests that AT Ara lies above the empirical and theoretical main sequence. This, however, can probably be explained by evolutionary effects. Figures 1 and 3 of Baraffe \& Kolb (2000) show clearly that the location of AT Ara secondary in the orbital period-spectral type plane cannot easily be reconciled with models of donor stars which were unevolved at the onset of mass transfer. However, there are no problems to explain its location assuming nuclear evolution of the donor prior to mass transfer. This implies a comparatively high initial mass $\left(\gtrsim 1 M_{\odot}\right)$. Considering the current low mass of $0.42 M_{\odot}$ of the AT Ara secondary and the modest mass of $0.53 M_{\odot}$ of the white dwarf, most of the transferred mass must have been expelled from the system in the course of the evolution.

\subsection{The distance to AT Ara}

The mean magnitude of AT Ara at quiescence is $V=14 .^{\mathrm{m}} 9$ (Bruch \& Engel 1994). To this value an error of 0. 3 is assigned which is half of the difference between the minimum and maximum magnitude difference between AT Ara and the principal comparison star in the five nights of differential photometry. Knowing the veiling factor and the size of the secondary star, it is then possible to calculate the distance to AT Ara.

For this purpose it is assumed that the spectrum of the primary component can be approximated by that of an infinite

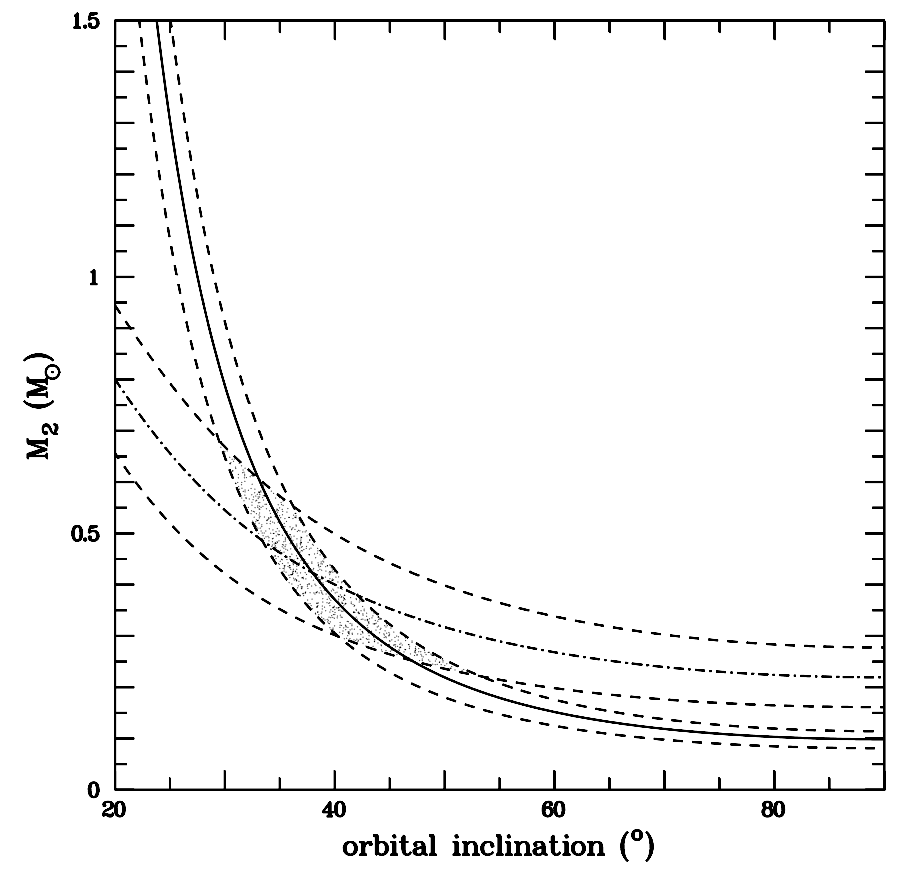

Fig. 10. Relation between the orbital inclination and the mass of the secondary star of AT Ara as derived from the projected rotational velocity (solid line), and the corresponding relation resulting from the total width of the $\mathrm{H} \alpha$ emission line (dashed-dotted line). The lateral dashed lines mark the error ranges for both relations.

Table 4. Dynamical and geometrical parameters of AT Ara.

\begin{tabular}{ll}
\hline \hline Orbital inclination $i$ (degrees): & $38 \pm 5$ \\
Primary star mass $M_{1}\left(M_{\odot}\right):$ & $0.53 \pm 0.14$ \\
Secondary star mass $M_{2}\left(M_{\odot}\right):$ & $0.42 \pm 0.10$ \\
Secondary star radius $R_{2}\left(R_{\odot}\right):$ & $0.84 \pm 0.19$ \\
\hline
\end{tabular}

steady state accretion disk (i.e. $F_{\lambda} \sim \lambda^{-7 / 3}$ ). Taking $V-R=$ 0.74 as the colour of a K2 main sequence star (Johnson 1966), and assuming a linear rise of the secondary star spectrum between the mean wavelengths of the $V$ and $R$ bands in order to determine its flux relative to that in the $V$ band at the wavelength where the veiling factor was measured, the veiling factor can be used to calculate the relative flux of the secondary star in the $V$ band. Knowing the apparent $V$ magnitude of the system, the $V$ magnitude of the secondary follows immediately. The absolute magnitude of a K2 V star is $\mathrm{M}_{V}=6.3$ according to a compilation based on HIPPARCOS results (Germano Quast, private comm.). Since the tables of Charbrier \& Baraffe (1997) do not contain a relation between mass and spectral type, a mass of $0.76 M_{\odot}$ for a K2 V star is taken from Schmidt-Kaler (1982). The 1 Gyr model of Charbrier \& Baraffe (1997) predict a radius of $0.72 R_{\odot}$ for a star of this mass. Considering the larger radius of the AT Ara secondary its absolute magnitude and then its distance module can be calculated.

With the above assumption about the spectrum of the primary component, the knowledge of the relative contributions of the components to the $V$ band and a colour index of $B-V=0.92$ for a K2 main sequence star (Johnson 1966), 
a colour index $(B-V)_{\mathrm{c}}=0.32 \pm 0.10$ for the entire system is predicted. Observations lead to a the mean colour index of $(B-V)_{\mathrm{o}}=0.74 \pm 0.10$ (Bruch 1983; Vogt 1983). Thus, the colour excess is $E_{B-V}=0.42 \pm 0$. 14 and, using a ratio of selective to total extinction of 3.15 , the visual extinction becomes $A_{V}=1.31 \pm 0.45$.

Another method to estimate the interstellar absorption possibly less fraught with uncertainties - consists in using the interstellar extinction map of Schlegel et al. (1998). At the location of AT Ara it yields $E_{B-V}=0.32$. Schlegel et al. (1998) cite an accuracy of their reddening values of the order of 0 m03 which is taken as the standard deviation here. Thus, $A_{V}=1.01 \pm 0{ }^{\mathrm{m}} 09$. Within their error margins, the independently derived extinction values coincide. However, since Schegel et al. measured the entire galactic extinction it should be regarded as an upper limit to the extinction of AT Ara. Even so, in view of the uncertainties inherent in the first method, the absorption based on the Schlegel et al. map is thought to be more reliable and is used in the calculations of the distance of AT Ara here (while distances based on the higher absorption are given in parentheses).

Putting everything together, a distance of $d=630 \pm 190 \mathrm{pc}$ $(d=550 \pm 190 \mathrm{pc})$ is derived for AT Ara. The error includes the uncertainties of the veiling factor, the apparent system magnitude in quiescent, the radius of the AT Ara secondary, and the observed colour index (and thus the interstellar extinction).

Using Warner's (1987) empirical relation between the maximum magnitude of dwarf novae in outburst and their orbital period, it is possible to derive an independent distance estimate. Since during outburst the overwhelming light source of a dwarf nova is the accretion disk the absolute magnitude should depend on the orbital inclination. Therefore, Warner's relation is based on magnitudes reduced to an average inclination. The same reduction, using the formula proposed by Paczyński \& Schwarzenberg-Czerny (1980), is applied here. This yields a difference between the true and the reduced magnitude of AT Ara of $\Delta m_{V}=0.59 \pm 0.11$. The error is due to the uncertainty of the orbital inclination. With this correction, and considering the errors of Warner's relation, the expected absolute outburst magnitude of a dwarf novae with the period of AT Ara should be $M_{V}=3.31 \pm 0.25$. The apparent magnitude of AT Ara during maximum is $m_{V}=12.61$ (Vogt 1983). An error of 0.1 is arbitrarily assigned to this value. The distance modulus is thus $m_{V}-M_{V}=9.89 \pm 0.29$ which together with the above mentioned interstellar extinction yields a distance of $d=610 \pm 90 \mathrm{pc}(d=520 \pm 130 \mathrm{pc})$. The two independently derived distance estimates thus show a very satisfying agreement.

\section{Conclusions}

The first time-resolved spectroscopic observations of the dwarf nova AT Ara confirmed earlier suspicions that the system is a double lined spectroscopic binary. Radial velocity curves have been determined for the $\mathrm{H} \alpha$ emission lines as well as for the absorption lines in the range $\lambda \lambda 5900-6500 \AA$. They reveal a comparatively long (for a cataclysmic variable) orbital period of almost exactly $9 \mathrm{~h}$. The amplitudes of the $R V$ curves suggest a mass ratio $q=0.79$.
The well developped absorption line spectrum allows a secure determination of the spectral class of the secondary star. The spectral type is $\mathrm{K} 2$, and there are no spectral features indicating a luminosity class higher than V. A detailed comparison between the AT Ara absorption line spectrum and the spectrum of a K2 V standard star yields a veiling factor of 0.55 and a rotational line broadening corresponding to $v_{\mathrm{r}} \sin i \approx 70 \mathrm{~km} \mathrm{~s}^{-1}$. The latter value can be used to derive an independent value of the mass ratio of $q=0.89$, consistent with the first value within the error margins.

A modest orbital inclination of $i=38^{\circ} \pm 5^{\circ}$ is derived which, in turn, yields component masses and the secondary star radius. The results show that its radius is considerably larger that that of a main sequence star with the same mass. According to the models of Baraffe \& Kolb (2000) this can probably be explained if the secondary star was already evolved at the onset of mass transfer, implying a fairly large initial mass of $>1 M_{\odot}$.

Acknowledgements. I thank U. Kolb for drawing my attention to the paper of Baraffe \& Kolb (2000). The wise comments of the referee, J. Thorstensen, have led to a decisive improvement of the paper. This research has made use of the SIMBAD database, operated at CDS, Strasbourg, France, and was partially supported by a grant of the Conselho Nacional de Desenvolvimento Científico e Tecnológico (CNPq; grant No. 301029).

\section{References}

Allard, F., Hauschildt, P. H., Alexander, P. R., \& Starrfield, S. 1997, ARA\&A, 35, 137

Anderson, J. 1991, A\&ARv, 3, 91

Baraffe, I., \& Kolb, U. 2000, MNRAS, 318, 354

Bateson, F. M. 1978, Publ. Var. Star Sect. RAS New Zealand, 6, 1

Beuermann, K., Baraffe, I., Kolb, U., \& Weichhold, M. 1998, A\&A, 339, 518

Bruch, A. 1982, Ph.D. Thesis, Münster

Bruch, A. 1983, IBVS, 2287

Bruch, A. 1993, MIRA - A Reference Guide (Universität Münster)

Bruch, A. 2000, A\&A, 359, 998

Bruch, A., \& Engel, A. 1994, A\&AS, 104, 79

Bruch, A., Steiner, J. E., \& Gneiding, C. D. 2000, PASP, 112, 237

Charbrier, G., \& Baraffe, I. 1997, A\&A, 327, 1039

Charbrier, G., Baraffe, I. 2000, ARA\&A, 38, 337

Deeming, T. J. 1975, Ap\&SS, 36, 137

Delfosse, X., Forveille, T., Mayor, M., Burnet, M., \& Perrier, C. 1999, A\&A, 341, L63

Eggleton, P. P. 1983, MNRAS, 268, 368

Friend, M. T., Martin, J. S., Smith, R. C., \& Jones, D. H. P. 1990, MNRAS, 246, 637

Hamuy, M., Walker, A. R., Suntzeff, N. B., et al. 1992, PASP, 104, 553

Harmanec, P. 1988, Bull. Astron. Inst. Czech. 39, 329

Honeycutt, K. R., Kaitchuck, R. H., \& Schlegel, E. M. 1987, ApJS, 65,451

Horne, K. 1986, PASP, 98, 609

Johnson, H. L. 1966, ARA\&A, 4, 193

Kolb, U., King, A. R., \& Baraffe, I. 2001, MNRAS, 321, 544

Metcalfe, T. S., Mathieu, R. D., Latham, D., \& Torres, G. 1996, ApJ, 456, 356

Nauenberg, M. 1971, ApJ, 175, 417 
Paczyński, B., \& Schwarzenberg-Czerny, A. 1980, Acta Astron. 30, 127

Popper, D. M. 1980, ARA\&A, 18, 115

Schlegel, D. J., Finkbeiner, D. P., \& Davis, M. 1998, ApJ, 500, 525

Schmidt-Kaler, T. 1982, in Landolt Börnstein, Numerical Data and

Functional Relationships in Science and Technology, ed. K.

Schaifers, \& H. H. Voigt, New Series, Group VI (Heidelberg: Springer Verlag), 2, 1

Schneider, D. P., \& Young, P. 1980, ApJ, 238946

Shafter, A. W. 1983, ApJ, 267, 222
Soubiran, C., Katz, D., \& Cayrel, R. 1998, A\&AS, 133, 221

Stehle, R., Ritter, H., \& Kolb, U. 1996, MNRAS, 279, 581

Vogt, N. 1976, in Structure and evolution of close binary systems, ed. P. Eggleton, S. Mitton, \& J. Whelan (Dordrecht: Reidel), Proc. IAU Symp., 73, 147

Vogt, N. 1983, A\&AS, 43, 21

Wade, R. A., \& Rucinski, S. M. 1985, A\&AS, 60, 471

Warner, B. 1976, in Multiple periodic variable stars, ed. W. S. Fitch, Proc. IAU Coll., 29, 247

Warner, B. 1987, MNRAS, 227, 23 